\title{
Reviewers of the current issue
}

\section{Prof. MA Taher}

Professor

Department of Radiology and Imaging

BIRDEM General Hospital, Dhaka, Bangladesh

\section{Prof. Rowshan Ara}

Professor

Department of Rheumatology

Green Life Medical College, Dhaka, Bangladesh

Dr. Ferdous Ur Rahman

Associate Professor

Department of Medicine

BSMMU, Dhaka, Bangladesh

\section{Dr. Jebun Nahar}

Associate Professor

Department of Paediatrics

BIRDEM General Hospital, Dhaka, Bangladesh

Dr. Md. Kamrul Hasan

Associate Professor

Department of Dermatology

BIRDEM General Hospital, Dhaka, Bangladesh

\section{Dr. Wasim Md. Mohosin UI Haque}

Associate Professor

Department of Nephrology

BIRDEM General Hospital, Dhaka, Bangladesh

\section{Dr. Abed Hossain Khan}

Assistant Professor

Department of Medicine

BSMMU, Dhaka, Bangladesh

\section{Dr. Farhana Afroz}

Assistant Professor

Department of Respiratory Medicine

BIRDEM General Hospital, Dhaka, Bangladesh

\section{Dr. Hasna Fahmima Haque}

Assistant Professor

Department of Internal Medicine

BIRDEM General Hospital, Dhaka, Bangladesh

\section{Dr. Md. Rashedul Islam}

Assistant Professor

Department of Neurology

BIRDEM General Hospital, Dhaka, Bangladesh

Dr. Tabassum Samad

Assistant Professor

Department of Nephrology

BIRDEM General Hospital, Dhaka, Bangladesh

Dr. Md. Jubaidul Islam

Registrar

Department of Internal Medicine

BIRDEM General Hospital, Dhaka, Bangladesh 